\author{
Wolfgang Zubke $\cdot$ Sven Becker $\cdot$ Bernhard Krämer • \\ Diethelm Wallwiener
}

\title{
Vaginal hysterectomy: a new approach using bicoagulation forceps
}

Published online: 3 June 2004

(C) Springer-Verlag Berlin / Heidelberg 2004

\begin{abstract}
This study is to assess the feasibility and outcome of vaginal hysterectomies using bicoagulation forceps. Eighty patients undergoing vaginal hysterectomy for several diagnoses were enrolled. In 40 patients, bicoagulation forceps were used for the entirety of the operation. In 19 patients, only vaginal hysterectomy was performed; in 21 patients, vaginal hysterectomy was part of surgery for pelvic floor repair. Forty control patients were operated on with the traditional technique. Data regarding intra- and postoperative complications, blood loss and duration of the hospital stay were compared. The intraand postoperative courses were similar in both groups. Intraoperative blood loss appeared less in the Biclamp group, particularly in the vaginal hysterectomy-only subgroup, as confirmed by pre- and postoperative hemoglobin measurements. Postoperative need for pain medication was significantly lower in the bicoagulation group, as was length of postoperative hospital stay. There was one postoperative bleeding complication requiring surgical revision 12 days after the initial surgery in the bicoagulation group. Using bicoagulation forceps without sutures for routine vaginal hysterectomy is a technically feasible and safe alternative to the traditional approach. Complication rates and patient satisfaction are similar. Blood loss and need for pain medication appear to be less in the coagulation group, leading to a reduced length of hospitalization.
\end{abstract}

Keywords Vaginal hysterectomy · Bicoagulation forceps - Surgical technique

\section{Introduction}

Vaginal hysterectomy is one of the most common surgical procedures in gynecology. In our institution, in 2003, 382

\footnotetext{
W. Zubke · S. Becker · B. Krämer $(\bowtie) \cdot$ D. Wallwiener Department of Obstetrics and Gynecology, University of Tuebingen Women's Hospital, Tuebingen, Germany e-mail: bernhardkraemer@gmx.de
}

hysterectomies for benign indications were performed, of which 161 were vaginal, 161 abdominal and 60 laparoscopic supracervical hysterectomies. Despite new treatment options $[1,2]$ developed for uterine disease over the past 3 decades such as hysteroscopy, laparoscopy and endocrine treatments, the number of all hysterectomies has not decreased significantly. Endoscopy has led to the development of new electrosurgical tools, particularly bicoagulation (BC) forceps that account for much of the success of the new technique. Vaginal hysterectomy is an established, "minimally invasive" approach. The basic technique has not been changed since the days of Stöckl, Kelly and Wertheim. We look at the technical and clinical feasibility and potential advantages of introducing the bicoagulation technique as a substitute for suturing in vaginal surgery.

\section{Material and methods}

For 12 months, the gynecologic surgeons of our institution have had access to the newly developed BiClamp bicoagulation forceps (Erbe, Tübingen, Germany). Using technologies originally developed for endoscopy, this tool was specially developed for vaginal surgery and corresponds to the traditional Haeney forceps (Fig. 1). The forceps are non-locking. In order to avoid irregular currents and accidental thermic injuries, they are covered with isolation material except for the areas specifically designed for coagulation. The electrical current is pulsatile and bipolar. Maximal strength is 4 A. The current is automatically controlled by the attached VIOErbe electrosurgical system, which leads to an automatic termination of the coagulation process. This allows for maximal hemostasis with minimal carbonization. Lateral damage to adjacent tissue is limited to an area of 1 to $2 \mathrm{~mm}$ of thermic denaturation. The BiClamp coagulation forceps are reusable.

The surgical technique using the bicoagulation forceps essentially follows the standard approach. The cervix is firmly grasped. The peritoneal cavity is entered anteriorly and posteriorly as usual, using a combination of sharp, blunt and electric dissection techniques. The sacrouterine ligaments are then grasped with the $\mathrm{BC}$ forceps, coagulated and cut without using any sutures. Then, following the established anatomic routine, the cardinal ligaments containing the uterine vessels are successively grasped, coagulated and cut using regular scissors. Again, no sutures are used. Depending on whether the adnexae are to be removed or left behind, either the infundibulopelvic or the ovarian and round ligaments are 


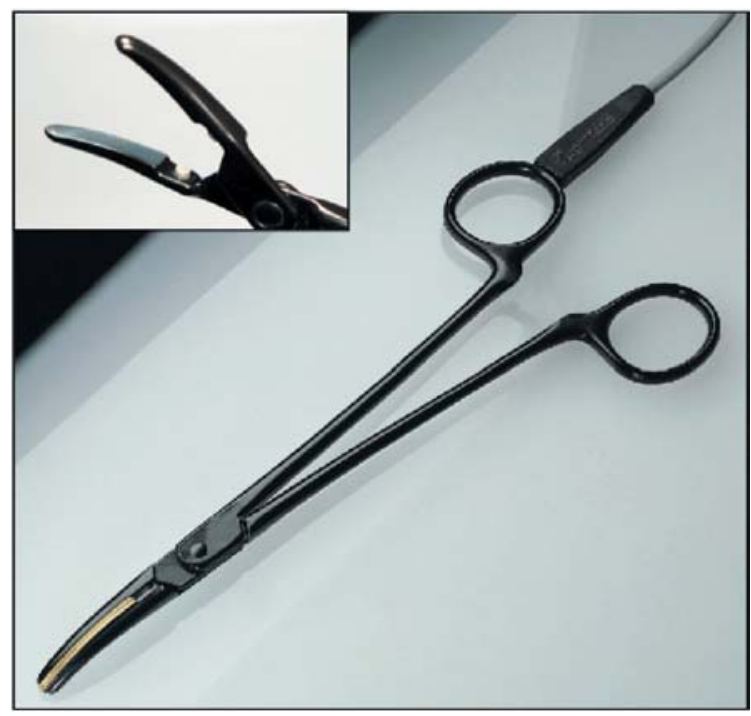

Fig. 1 BiClamp bicoagulation forceps (Erbe, Tübingen, Germany). They are covered with isolation material except for the areas specifically designed for coagulation

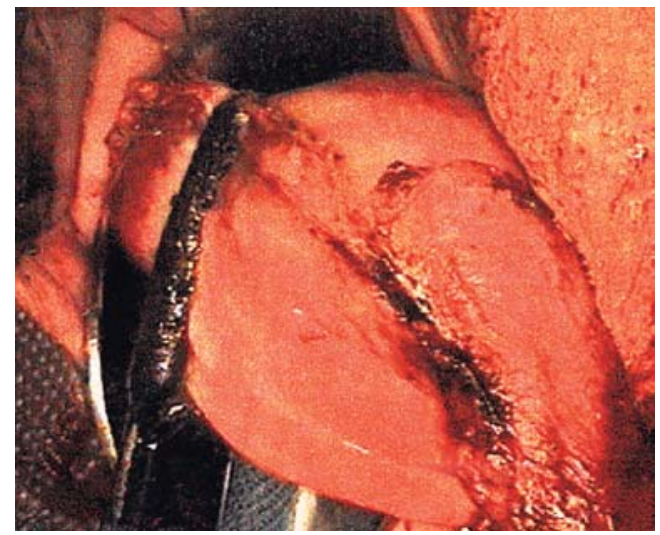

Fig. 2 Either the infundibulopelvic or the ovarian and round ligaments are coagulated and cut

grasped, coagulated and cut (Fig. 2). After removal of the uterus, the vaginal cuff is closed using continuous suture. A combination with other closure and suspension techniques such as McCall culdoplasty is possible without difficulty.

In this prospective study, we compare 40 vaginal hysterectomies using BiClamp with 40 similar vaginal cases using the traditional technique. All operations were performed by the same surgeon. Looking at the BiClamp group, 19 patients underwent vaginal hysterectomy only, and 21 patients underwent more extensive pelvic floor reconstruction, of which vaginal hysterectomy was one aspect (Table 1). The comparison group was similarly divided into hysterectomy only and hysterectomy as part of pelvic reconstruction. Table 2 summarizes the main patient characteristics from both groups. Both groups were similar with regard to medical history, surgical risk factors and indications for surgery. All patients received preoperative antibiotic therapy. During the entire hospital stay, all patients received prophylactic low molecular weight heparin.

\section{Results}

No major intraoperative complications occurred in either group. Particularly, no injury to adjacent organs of the pelvis occurred. Blood transfusions or discontinuation of the vaginal approach and switching to an abdominal approach were not necessary. Coagulation using the bicoagulation forceps provided adequate lateral hemostasis in almost all cases. In three patients only, additional sutures were required to secure suboptimally hemostatic pedicles.

The estimated intraoperative blood loss was $107 \mathrm{ml}$ (range 5-400 ml) in the BiClamp group, which was slightly less than in the control group (148 ml, range $30-400 \mathrm{ml}$ ). The postoperative drop in hemoglobin was $1.5 \mathrm{~g} / \mathrm{dl}(0-5.8 \mathrm{~g} / \mathrm{dl})$ in the Biclamp group and $1.6 \mathrm{~g} / \mathrm{dl}$ $(0-5.6 \mathrm{~g} / \mathrm{dl})$ in the control group (Table 3$)$. These differences were more pronounced in the vaginal hysterectomy only group with an estimated blood loss of $59 \mathrm{ml}$ compared to $100 \mathrm{ml}$ and postoperative hemoglobin drop of $1.0 \mathrm{~g} / \mathrm{dl}$ versus $1.3 \mathrm{~g} / \mathrm{dl}(P<0.05)$ (Table 4$)$. In three patients of the BiClamp group, additional sutures were required after coagulation of the vessel-containing pedicles for extra safety.

With regard to pain medication requirements, $24 \mathrm{Bi}-$ Clamp patients versus 32 control patients required 107 versus 132 applications of pain medication (Table 5, Table 6). Again, looking at the subgroup of hysterectomy-

Table 1 Different procedures performed both in the BiClamp and in the control group

\begin{tabular}{llc}
\hline Operation & BiClamp & Control \\
\hline No. of patients & 40 & 40 \\
Pure vaginal hysterectomy & 19 & 19 \\
Vaginal adnexectomy & 2 & 2 \\
Midline cystocele repair & 14 & 15 \\
Rectocele repair & 11 & 10 \\
Sacrospinous ligament suspension & 6 & 8 \\
Tension-free vaginal tape (TVT) & 9 & 7 \\
McCall culdeplasty & 3 & 2 \\
Part. colpectomy & 1 & 1 \\
Vaginal paravag. repair & 2 & 2 \\
Mesh implantation & 0 & 1 \\
\hline
\end{tabular}

Table 2 Patient characteristics and median weight of the removed uterus of both groups

\begin{tabular}{lcc}
\hline Patients & BiClamp & Control \\
\hline No. & 40 & 40 \\
Age (years) & $52.9(32-87)$ & $53.9(36-80)$ \\
Weight $(\mathrm{kg})$ & $75.8(36-132)$ & $75.2(48-120)$ \\
Uterus $(\mathrm{g})$ & $140(35-360)$ & $145(35-840)$ \\
\hline
\end{tabular}

Table 3 Estimated intraoperative blood loss and postoperative drop in hemoglobin in both groups

\begin{tabular}{lcc}
\hline & BiClamp & Control \\
\hline No. (all) & 40 & 40 \\
Valued $(\mathrm{ml})$ & $107(5-400)$ & $148(30-400)$ \\
Drop of $\mathrm{Hb}(\mathrm{g} / \mathrm{dl})$ & $1.56(0-5.8)$ & $1.64(0-5.6)$ \\
\hline
\end{tabular}


Table 4 Vaginal hysterectomy-only group: the difference in postoperative hemoglobin drop is significant $(P<0.05)$

\begin{tabular}{lll}
\hline & BiClamp & Control \\
\hline No. (pure vag. hyst.) & 19 & 19 \\
Valued (ml) & $58.6(5-150)$ & $99.5(50-400)$ \\
Drop of Hb $(\mathrm{g} / \mathrm{dl})$ & $1.0(0-2.5)$ & $1.3(0.3-2.8)$ \\
\hline
\end{tabular}

Table 5 Pain medication in both groups after the 1st postoperative day

\begin{tabular}{lcc}
\hline & BiClamp & Control \\
\hline No. (all) & 40 & 40 \\
Patient no. & 24 & 32 \\
Single doses & 107 & 132 \\
\hline
\end{tabular}

Table 6 Pain medication in the hysterectomy-only subgroup: the number of patients who required pain medication and the number of single doses varied significantly $(P<0.01)$

\begin{tabular}{lll}
\hline & BiClamp & Control \\
\hline No. (pure vaginal hysterectomy) & 19 & 19 \\
Patient no. & 9 & 19 \\
Single doses & 26 & 94
\end{tabular}

Table 7 Postoperative hospital stay for the whole group

\begin{tabular}{lll}
\hline & BiClamp & Control \\
\hline No. (all) & 40 & 40 \\
Days & $6.6(3-12)$ & $7.4(4-14)$ \\
\hline
\end{tabular}

Table 8 Hospital stay for the vaginal hysterectomy-only group $(P<0.05)$

\begin{tabular}{lll}
\hline & BiClamp & Control \\
\hline No. ( vaginal-hysterectomy only) & 19 & 19 \\
Days & $5.8(3-11)$ & $7.1(5-10)$ \\
\hline
\end{tabular}

only patients, this difference becomes more clearly in favor of the bicoagulation group: 9/19 patients required a total of 26 pain medication applications versus 19/19 patients requiring a total of 94 pain medication applications $(P<0.01)$.

The postoperative hospital stay was 6.6 days (3-11 days) in the bicoagulation group versus 7.4 days in the control group (4-14 days). For the vaginal hysterectomyonly group, these times were 5.8 days $(3-11)$ versus 7.1 days $(5-10$ days), $(P<0.05)$, (Table 7 , Table 8$)$. During the postoperative period, no complications such as fever, urinary tract infections or other problems occurred in either group. However, one patient from the Biclamp group had to be readmitted on postoperative day 12 for new onset vaginal bleeding. The patient had to be taken to the operating theater, where a small bleeding parametrian vessel was identified and ligated without problems.
Again, no blood transfusion was necessary. It remains unclear whether this was random or related to our method of coagulation. It did, however, lead us to change the modulation to provide a more continuous transition zone from necrotic to healthy tissue. Most likely, the bleeding episode was secondary to shedding of the area of necrosis.

In our institution, the routine intraoperative use of the BiClamp bicoagulation forceps was possible without any major difficulties. Forty hysterectomies were performed with this technique, and results were as good as or better than with the traditional technique of clamping, cutting and ligating the pedicles of the parametrian tissue $[3,4]$. As with the laparoscopic approaches $[5,6,7,8]$, various points need to be observed with the new technique: during coagulation, no tension or pulling must occur, otherwise only partially coagulated tissue could tear off, leading to bleeding. Occasionally, subsequent and neighboring areas of tissue were coagulated before being cut. Particular care has to be used with edematous tissue, as the electrical current will vaporize the tissue water, leading to tearing of the tissue immediately adjacent to the clamp. To avoid accidental injuries to neighboring tissue and organs, a safety distance of 5 to $10 \mathrm{~mm}$ was meticulously observed.

During our experience with the bicoagulation forceps, it became clear that particularly difficult vaginal cases were easier to perform with the Biclamp. As no ligatures and sutures were required, space requirements were less. Also, small "bites" could be taken quickly, one after another, something that cannot be done with sutures. Finally, sutures tend to decrease visibility of the surgical field, which does not happen with the bicoagulation forceps. Only rarely did it become necessary to apply additional ligatures when we felt that the hemostasis achieved with coagulation alone was not sufficient. While the bicoagulation forces were used in pelvic reconstructive cases, their application was strictly limited to the vaginal hysterectomy part. While the technique is basically easy, there is a learning curve that should be observed. We suggest a training period with an experienced surgeon familiar with the BiClamp technique.

Looking at important clinic parameters such as blood loss and pain medication requirements, the bicoagulation technique appeared as good as, if not better than, the traditional approach. Bicoagulation not only provides hemostasis with regard to the lateral pelvic sidewall, but also avoids retrograde bleeding from the uterus, whichparticularly in the case of large uteri-can be considerable and is often the most important source of bleeding during vaginal hysterectomies. This main advantage leads to an almost perfect hemostatic surgery (Fig. 3). With regard to postoperative pain, the theory is that coagulation destroys nerve endings rather than involving them in sutured pedicles where tension and stretching lead to an increased pain sensation by the patient. Other surgeons, in particular Clavé, have described similar results [9]. While these reports report a reduced operative time, in our impression, the total length of our procedures was not significantly altered. We did not include this data because many of our cases were part of in-house teaching. 


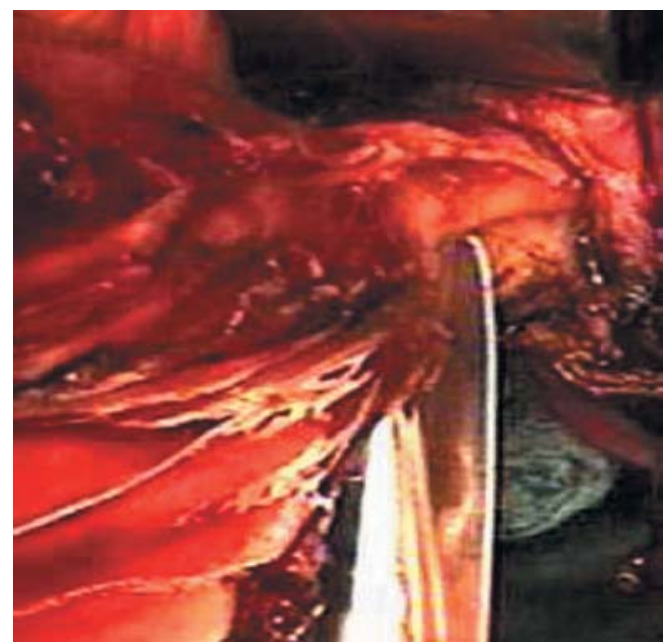

Fig. 3 Coagulation and cutting of the vessels of the adnexae by almost perfect hemostatic surgery

With reduced postoperative pain requirements, less need for sutures and shortened postoperative stay, important economic arguments could be raised for this new approach. While we observed one unusual complication out of 40 cases $(2.5 \%)$, we feel that the BiClamp bicoagulation forceps offer a safe and interesting new concept to vaginal hysterectomies that can be combined with other vaginal operations, for example, the insertion of a tensionfree vaginal tape (TVT) and pelvic floor surgery [10].

\section{References}

1. Loh FH, Koa RC (2002) Laparoscopic hysterectomy versus abdominal hysterectomy: a controlled study of clinical and functional outcomes. Singapore Med J 43:403-407

2. Wallwiener D, Aydeniz B, Rimbach S et al (1996) Der Wandel des Spektrums uteruserhaltender Myomoperationen unter Einbeziehung von Endoskopie und dualer Myomtherapie. Gynäkol Geburtsh Rundsch 36:118-132

3. Karram MM (2001) Vaginal hysterectomy. In: Baggish MS, Karram MM (2001) Atlas of pelvic anatomy and gynecologic surgery. WB Saunders, Philadelphia

4. Thompson JD. Thompson JD, Rock JA (1992) Te Linde's operative gynecology, 17th edn. Lippincott, Philadelphia

5. Liu CY (1992) Laparoscopic hysterectomy. A review of 72 cases. J Reprod Med 37:351-354

6. Dubuc-Lissoir J (2003) Use of a new energy-based vessel ligation device during laparoscopic gynecologic oncologic surgery. Surg Endosc 17:466-468

7. Landman J, Kerbl K, Rehman J et al (2003) Evaluation of a vessel sealing system, bipolar electrosurgery, harmonic scalpel, titanium clips, endoscopic gastrointestinal anastomosis vascular staples and sutures for arterial and venous ligation in a porcine model. J Urol 169:697-700

8. Heniford BT, Matthews BD, Sing RF, Backus C, Pratt B, Green FL (2001) Initial results with an electrothermal bipolar vessel sealer. Surg Endosc 15:799-801

9. Clavé H, Niccolai P (2003) Hysterectomie sans douleurs: une technique innovante. J Gynecol Obstet Biol Reprod (Paris) 32:375-380

10. Zubke W, Schröter M, Wallwiener D (2001) TVT zum Vorgehen in der Praxis. Geburtsh Frauenheilk 61:426-429 